\title{
Determination of discriminatory traits in maize hybrids
}

\author{
Carlos B. BANCHERO ${ }^{a *}$, Norberto BARTOLONI $^{\mathrm{a}}$, Miguel Angel RAPELA ${ }^{\mathrm{b}}$, \\ Gabriel MARRASSINI ${ }^{\mathrm{a}}$, Santiago RENTERÍA ${ }^{\mathrm{a}}$ \\ ${ }^{a}$ Cátedra de Genética, Facultad de Agronomía, Universidad de Buenos Aires, Av. San Martín 4453 (1417), Buenos Aires, Argentina \\ b Agar Cross S.A., División Semillas, Rosario, Santa Fe, Argentina
}

(Received 1 April; accepted 9 June 2000)

\begin{abstract}
A discriminant analysis was performed on a set of morphological characteristics for a group of Argentine commercial maize (Zea mays L.) hybrids. In the discriminant analysis a set of canonical varieties was constructed to assess the discriminatory incidence of those characteristics among commercial hybrids. Discriminant analyses were performed on plant, ear and kernel characters. With the exception of whole plant traits, significant discriminations were found and the composition of the canonical variables explaining them was determined. For traits measured on the whole plant, ear insertion height was primarily responsible for the variation, and a noticeable level of uniformity was observed with respect to stalk diameters. Ear traits (ear medium diameter and ear length), kernel weight and number of kernels, exhibited the most significant discrimination among hybrids. Finally, with respect to kernel traits, significant distances among hybrids were detected, and kernel length and kernel breadth were the most important traits.
\end{abstract}

maize / hybrids / discriminant analysis / breeding / variability

Résumé - Détermination de caractères discriminants dans des hybrides de maïs. Une analyse discriminante a été faite pour un ensemble de caractères morphologiques chez un groupe d'hybrides commerciaux de maïs argentins (Zea mays L.). Avec cette analyse, une série de variables canoniques a été définie pour évaluer la capacité discriminante de ces caractères entre les hybrides. Des analyses discriminantes ont été réalisées pour des caractères de plante entière, de l'épi et du grain. À l'exception des caractères correspondants à la plante entière, des discriminations significatives ont été trouvées pour tous les caractères étudiés et la composition des variables canoniques pour les expliquer a été définie. Entre les caractères mesurés pour la plante entière, la hauteur d'insertion de l'épi a été le principal responsable de la variation, tandis que le diamètre de la tige a été le plus stable. Les caractères mesurés pour l'épi (diamètre moyen et longueur), le poids du grain et le nombre de grains ont été les plus significatives entre les hybrides. Finalement, des distances significatives entre hybrides ont été détectées avec la longueur et la largeur du grain.

maïs / hybrides / analyse discriminante / amélioration de plantes / variabilité

Communicated by Max Rives (Villeneuve-lès-Avignon, France)

* Correspondence and reprints

banchero@mail.agro.uba.ar 


\section{Introduction}

The importance of genetic variability in commercial breeding has been repeatedly emphasized [9] as an essential requirement for the improvement of traits associated with crop production and quality. Thus, it would be interesting to know which traits show discrimination among populations such as commercial hybrids in order to use them in the outlining of breeding programs. Examples of the consequences of lacking such variability have been documented [1].

Assessing genetic variability has been greatly improved with the use of multivariate analysis techniques $[14,15]$. Since the expansion of computers, their use has been greatly extended through a vast array of scientific as well as technological areas. In biology, applications such as numerical taxonomy, cladistics, and evolution are widely known $[3,8,10,11]$.

The variability of populations under breeding processes has been assessed in different species and environments [2,4-6]. Our objective in this work was to determine whether there is any degree of statistically significant discrimination among maize (Zea mays) hybrids in current commercial production in Argentina and which traits most contribute to it. To do this, we applied discriminant analysis [15] to macroscopic traits of agronomic interest in some Argentine commercial hybrids. Discriminant functions allow the breeders to look over one aspect of the critical issue of variability inside commercial populations. This was possible with the collaboration of three international corn breeding companies that work in Argentina, namely: Dekalb Argentina SA, Pioneer Argentina SA and Cargill Argentina SACIF. These companies provided seeds from their most representative corn hybrids. Discriminant analysis allows us to get a general picture of differences eliminating the correlations that obscure the readings.

It has been frequently discussed whether the residual variability within the commercial populations of the most important plant species is currently under the threat of being lost given its dramatic reduction due to the utilization of a very narrow genetic base and the subsequent breeding process [12]. Many corn hybrids currently under production are based on inbred lines that have a common germplasm. Under this assumption, it is expected that the hybrids were closely related and low discrimination would be found among their traits. Here we have tested such an hypothesis, i.e. that the discrimination among several traits is not statistically significant.

We have calculated canonical variates, which allow the reduction in data dimensions and the inference of a biological interpretation from the overall variation in the experiment. Then, if there were any degree of discrimination among the groups being surveyed, it might be summarized in a few variates.

\section{Materials and methods}

\subsection{Genetic materials}

The following fifteen hybrids from a sample provided by the companies were tested:

Dekalb Argentina SA: 638, 664, 752 (singlecross hybrids $[2 \mathrm{X}]$ ), 3F24, 3S41 (triple-cross hybrids $[3 \mathrm{X}]$ ) and 4F37, 4F91, 762 (double-cross hybrids[4X]).

Pioneer Argentina SA: 3456, 3162 (single-cross hybrids[2X]) and 3478 (triple-cross hybrid[3X]).

Cargill Argentina SA: M31, TH92, T327 (triplecross hybrids[3X]) and SD5 (double-cross hybrids[4X]).

\subsection{Experimental design}

The experiment was developed in the corn nursery of the Facultad de Agronomía. A Randomized Complete Block Design was used. The experiment comprised 300 plants (20 plants per hybrid), in 2 complete blocks, with 10 plants (replicates) per block, i.e. 10 plants in a row with $0.70 \mathrm{~m}$ between rows. The groups (hybrids) were randomly distributed in the blocks. Data from individual plants were analyzed. A multivariate analysis of variance 
(MANOVA) [15] among the hybrids for all the traits measured was calculated. Thus, there has been in the MANOVA a HYBRID term, a BLOCK term, an ERROR term and an INTERACTION $($ BLOCK $\times$ REPLICATE) term. There was no significance in the $\mathrm{F}$ Snedecor for the interaction term, nor for the blocks (probably due to the uniform environment in the nursery).

\subsection{Morphological measurements}

Measurements were taken on plant, ear, and kernels in the Facultad de Agronomía (Universidad de Buenos Aires) in 1996 and 1997.

(1) Measurements on plants: plant height $(\mathrm{PH})$ in $\mathrm{cm}$, lower major stalk diameter (DIAM2L) in $\mathrm{cm}$, lower minor stalk diameter (DIAM2C) in $\mathrm{cm}$, upper major stalk diameter (DIAM1L) in $\mathrm{cm}$, upper minor stalk diameter (DIAM1C) in $\mathrm{cm}$, and ear insertion height (EIH) in $\mathrm{cm}$ (the lower ear, where there were more than one); lower diameters were taken at the base of the stalk, while the upper ones were measured on the stalk upper portion above the ear. One ear per plant was measured.

(2) Measurements on ears: Ear architecture: ear length (EL) in $\mathrm{cm}$, ear basal diameter (EBD) in $\mathrm{cm}$ and ear medium diameter (EMD) in $\mathrm{cm}$; Measurements associated with yield: husk weight $(\mathrm{HW})$ in $\mathrm{g}$, number of husks $(\mathrm{NH})$, mean number of kernels per row (NKR) (30 kernels per ear were sampled), kernel weight (KW) in $\mathrm{g}$, cob weight $(\mathrm{CW})$ in $\mathrm{g}$, number of rows (NR) and total number of kernels (NK).

(3) Measurements on kernels: kernel breadth $(\mathrm{KB})$ in $\mathrm{cm}$, kernel length (KL) in $\mathrm{cm}$ and kernel thickness (KT) in $\mathrm{cm}$.

\subsection{Canonical analysis}

To statistically test the degree and nature of discrimination among the groups of hybrids, canonical variates $[3,16]$ accounting for most of the variation of the experiment were obtained (see appendix).

The discriminant analysis was performed with this canonical analysis providing a set of new vari- ables extracted from the data. The original data matrix Y was transformed (see appendix) to obtain a set of fully non-correlated variables that maximize the variation among the groups while minimizing variation within them. Such canonical variates are linear combinations of the original ones, and they were standardized to make comparisons across them. Also, the corresponding $\chi^{2}$ tests for each of the successive roots extracted were computed. Analyses were performed separately for each type of hybrid (whether $2 \mathrm{X}, 3 \mathrm{X}$ or $4 \mathrm{X}$ ) computing for each case:

(a) Wilks' $\lambda$ significance tests to assess the global degree of discrimination;

(b) $\chi^{2}$ significance tests to calculate the number of canonical roots;

(c) Standardized coefficients constituting each canonical root.

\section{Results}

\subsection{Plant architecture traits}

The general discriminant analysis corresponding to plant architecture characteristics shows a notable difference among types of hybrids. For the $2 \mathrm{X}$ hybrids there is significant discrimination $(p<0.05)$ for 3 traits (PH, DIAM2C and EIH) (Tab. I). In turn, for $3 \mathrm{X}$ and $4 \mathrm{X}$ hybrids there seems to be a highly significant discrimination with respect to EIH and, in the case of double hybrids, also to PH.

The multivariate data analysis allowed the reduction of the multidimensional array of points to a tri-, uni- and univariate distribution for the $2 \mathrm{X}$, $3 \mathrm{X}$, and $4 \mathrm{X}$ hybrids, respectively (Tab. II) using a $\chi^{2}$ probability criterion (i.e. $p<0.01$ ): 3 significant canonical roots were extracted for the $2 \mathrm{X}$ group, and only 1 root for $3 \mathrm{X}$ and $4 \mathrm{X}$ hybrids.

The composition of those canonical roots in terms of the original variables is shown in Table III. In all the 3 groups it was clear that the main component of the first canonical root (the most important in all cases) was EIH with an explanatory power greater than $61 \%$ in the case of $2 \mathrm{X}$ hybrids, greater than $90 \%$ for $3 \mathrm{X}$ hybrids, and 
greater than $84 \%$ in the case of double hybrids. In agreement with what was explained when commenting in Table II, only 1 canonical variate is nec-

Table I. Discriminant analysis with $\lambda$ Wilks tests corresponding to measurements taken on the whole plant*.

\begin{tabular}{llll}
\hline Hybrid Type & Variable & Wilks $\lambda$ & $p<$ \\
\hline $2 \mathrm{X}$ & PH & 0.311 & 0.0040 \\
& DIAM1C & 0.287 & 0.0931 \\
& DIAM2C & 0.327 & 0.0005 \\
& DIAM1L & 0.292 & 0.0516 \\
& DIAM2L & 0.272 & 0.5299 \\
$3 \mathrm{X}$ & EIH & 0.440 & 0.0001 \\
& PH & 0.201 & 0.2593 \\
& DIAM1C & 0.198 & 0.4694 \\
& DIAM2C & 0.195 & 0.7190 \\
& DIAM1L & 0.203 & 0.1736 \\
$4 \mathrm{X}$ & DIAM2L & 0.207 & 0.0813 \\
& EIH & 0.385 & 0.0001 \\
& PH & 0.371 & 0.0002 \\
& DIAM1C & 0.285 & 0.9028 \\
& DIAM2C & 0.286 & 0.7994 \\
& DIAM1L & 0.287 & 0.7484 \\
& DIAM2L & 0.290 & 0.5763 \\
& EIH & 0.574 & 0.0001 \\
\hline
\end{tabular}

* PH: plant height, DIAM1C: upper minor stalk diameter, DIAM2C: lower minor stalk diameter, DIAM1L: upper major stalk diameter, DIAM2L: lower major stalk diameter, EIH: ear insertion height.

Table II. Canonical roots extracted and $\chi^{2}$ tests corresponding to measurements taken on plants.

\begin{tabular}{lccccc}
\hline Hybrid type Roots & Eigenvalue & $\chi^{2}$ & D. F. & $p<$ \\
\hline $2 \mathrm{X}$ & 1 & 1.091 & 124.869 & 24 & 0.0001 \\
& 2 & 0.404 & 55.894 & 15 & 0.0001 \\
& 3 & 0.191 & 24.188 & 8 & 0.0021 \\
& 4 & 0.088 & 7.849 & 3 & 0.0493 \\
$3 \mathrm{X}$ & 1 & 2.991 & 187.884 & 30 & 0.0001 \\
& 2 & 0.219 & 31.480 & 20 & 0.0493 \\
& 3 & 0.079 & 9.109 & 12 & 0.6936 \\
& 4 & 0.004 & 0.504 & 6 & 0.9978 \\
$4 \mathrm{X}$ & 5 & 0.001 & 0.103 & 2 & 0.9500 \\
& 1 & 1.634 & 93.361 & 18 & 0.0001 \\
& 2 & 0.229 & 21.320 & 10 & 0.0190 \\
& 3 & 0.085 & 6.071 & 4 & 0.1940 \\
\hline
\end{tabular}

essary to account for the discrimination for $3 \mathrm{X}$ and $4 \mathrm{X}$ hybrids and $2(84.29 \%)$ or $3(95.06 \%)$ for $2 X$ ones. The second canonical variate corresponding to $2 \mathrm{X}$ hybrids is constituted mainly by DIAM2C.

The great explanatory power of the 2 first canonical roots is clearly shown. We have included the second canonical root in the case of $2 \mathrm{X}$ hybrids because it explains almost $23 \%$ of the total variation, but it is worth taking into account its low eigenvalue $(0.404$, less than half of the recommended value of 1). Hence, EIH seems to be the main reason why groups are different regarding the whole plant traits analyzed in this experiment.

\subsection{Ear architecture traits}

Table IV shows the discriminant analysis abstract corresponding to ear architecture traits. Clearly, EBD does not contribute to much of the discrimination, among $2 \mathrm{X}$ hybrids and among $4 \mathrm{X}$ ones. In the case of the $2 \mathrm{X}$ hybrids EL and EMD determine a highly significant discrimination among hybrids while in the case of $3 \mathrm{X}$ groups, EL and EBD do. Also for the 4X hybrids EL and EMD are the main discriminatory variables but to a lesser significance level.

As in the case of plant traits, 2 of the extracted roots were highly significant $\left(p\left[\chi^{2}\right]<0.01\right)$ in the case of $2 X$ hybrids but only one in the other cases (Tab. V). The composition of the canonical variables shows a clear picture for single hybrids in which case the first canonical root explains more than $92 \%$ of total variation, this root being constituted mainly by EMD. Discrimination among $3 \mathrm{X}$ hybrids is explained mainly by a sort of "ear shape" coefficient $(-0.739 \cdot \mathrm{EL}+0.898 \cdot \mathrm{EBD})$. Among 4X hybrids, discrimination is accounted for by an "ear size" coefficient (0.634.EBD + 0.800.EMD) (Tab. VI).

\subsection{Ear measurements associated with yield}

The overall discriminant analysis shows a complex pattern in terms of the original variables (Tab. VII). In order to summarize it, it may be said that there are no statistically significant differences 
Table III. Standardized coefficients for each canonical variate corresponding to measurements taken on plants*.

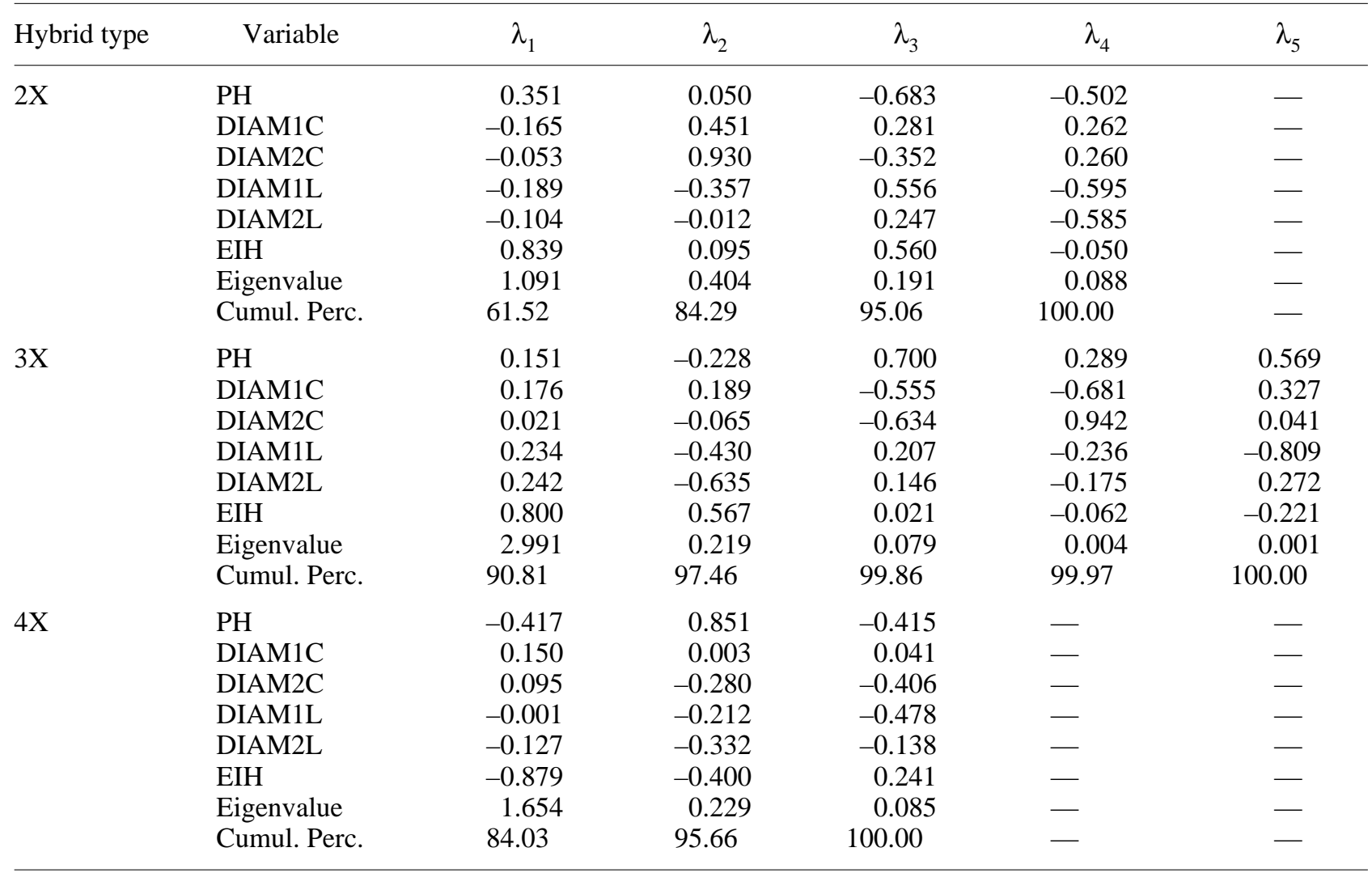

* PH: plant height, DIAM1C: upper minor stalk diameter, DIAM2C: lower minor stalk diameter, DIAM1L: upper major stalk diameter, DIAM2L: lower major stalk diameter, EIH: ear insertion height.

Table IV. Discriminant analysis with $\lambda$ Wilks tests corresponding to ear architecture traits*.

\begin{tabular}{llcc}
\hline Hybrid type & Variable & Wilks $\lambda$ & $p<$ \\
\hline $2 X$ & EL & 0.393 & 0.0001 \\
& EBD & 0.279 & 0.1383 \\
& EMD & 0.778 & 0.0001 \\
$3 \mathrm{X}$ & EL & 0.776 & 0.0099 \\
& EBD & 0.818 & 0.0008 \\
$4 \mathrm{X}$ & EMD & 0.764 & 0.0208 \\
& EL & 0.813 & 0.0133 \\
& EBD & 0.760 & 0.1257 \\
& EMD & 0.800 & 0.0226
\end{tabular}

* EL: ear length, EBD: ear basal diameter, EMD: ear medium diameter.
Table V. Canonical roots extracted and $\chi^{2}$ tests corresponding to ear architecture traits.

\begin{tabular}{lccccc}
\hline Hybrid type Roots & Eigenvalue & $\chi^{2}$ & D. F. & $p<$ \\
\hline $2 \mathrm{X}$ & 1 & 2.225 & 128.205 & 12 & 0.0001 \\
& 2 & 0.115 & 16.966 & 6 & 0.0094 \\
& 3 & 0.072 & 6.631 & 2 & 0.0363 \\
$3 \mathrm{X}$ & 1 & 0.242 & 44.262 & 15 & 0.0001 \\
& 2 & 0.124 & 19.440 & 8 & 0.0127 \\
$4 \mathrm{X}$ & 3 & 0.054 & 6.003 & 3 & 0.1115 \\
& 1 & 0.227 & 26.297 & 9 & 0.0018 \\
& 2 & 0.158 & 11.037 & 4 & 0.0262 \\
& 3 & 0.002 & 0.128 & 1 & 0.7201 \\
\hline
\end{tabular}


Table VI. Standardized coefficients for each canonical variate corresponding to ear architecture traits*.

\begin{tabular}{llccc}
\hline Hybrid type & Variable & $\lambda_{1}$ & $\lambda_{2}$ & $\lambda_{3}$ \\
\hline $2 \mathrm{X}$ & EL & 0.839 & -1.017 & -0.0019 \\
& EBD & 0.080 & -0.089 & 1.035 \\
& EMD & -1.334 & 0.044 & -0.260 \\
& Eigenvalue & 2.225 & 0.115 & 0.072 \\
& Cumul. Perc. & 92.24 & 97.00 & 100.00 \\
$3 \mathrm{X}$ & EL & -0.739 & 0.633 & 0.666 \\
& EBD & 0.898 & 0.439 & 0.278 \\
& EMD & 0.280 & -1.083 & 0.340 \\
& Eigenvalue & 0.242 & 0.125 & 0.054 \\
$4 \mathrm{X}$ & Cumul. Perc. & 58.58 & 87.20 & 100.00 \\
& EL & -0.043 & -1.096 & -0.059 \\
& EBD & 0.634 & 0.015 & 0.778 \\
& EMD & 0.800 & 0.449 & -0.599 \\
& Eigenvalue & 0.227 & 0.158 & 0.002 \\
& Cumul. Perc. & 58.78 & 99.55 & 100.00
\end{tabular}

* EL: ear length, EBD: ear basal diameter, EMD: ear medium diameter.

among $2 \mathrm{X}$ and $3 \mathrm{X}$ hybrids with regard to NKR and $\mathrm{NK}$ and that, with the exception of $\mathrm{NH}$ and NK, all variables show differences among $4 \mathrm{X}$ hybrids. $3 \mathrm{X}$ hybrids show a lesser degree of discrimination among them. The $\chi^{2}$ tests shown in Table VIII and the canonical variates constitution in Table IX indicate that at least 2 of the roots should be taken in mind in order to explain most of the variation. In the case of $2 X$ hybrids they explain $88.7 \%$ of the total variation, while for $3 \mathrm{X}$ hybrids almost $80 \%$ is explained. In the case of double hybrids the first 2 canonical roots explain $94 \%$. With respect to the root components, KW (1st root) and NK (2nd root) are the main components in the case of $2 \mathrm{X}$ hybrids. HW (1st root) and a linear combination of the kernel numbers $(-2.798 \cdot \mathrm{NKR}+2.523 \cdot \mathrm{NK})$ are the main components in the case of $3 \mathrm{X}$ hybrids. Finally, NKR and NK are the principal root components in the case of $4 \mathrm{X}$ hybrids.

\subsection{Kernel traits}

The main character is KL while KT seems to make little or no contribution in all three groups
Table VII. Discriminant analysis with $\lambda$ Wilks tests and corresponding to ear measurements associated with yield*.

\begin{tabular}{llcc}
\hline Hybrid type & Variable & Wilks $\lambda$ & $p<$ \\
\hline $2 \mathrm{X}$ & HW & 0.060 & 0.0005 \\
& NKR & 0.050 & 0.2767 \\
& KW & 0.091 & 0.0001 \\
& NH & 0.081 & 0.0001 \\
& NR & 0.056 & 0.0079 \\
& NK & 0.052 & 0.0670 \\
& CW & 0.059 & 0.0010 \\
$3 X$ & HW & 0.289 & 0.0001 \\
& NKR & 0.185 & 0.4373 \\
& KW & 0.195 & 0.0586 \\
& NH & 0.245 & 0.0001 \\
NR & 0.190 & 0.1825 \\
& NK & 0.186 & 0.3813 \\
$4 \mathrm{CW}$ & 0.214 & 0.0010 \\
& HW & 0.3108 & 0.1231 \\
& NKR & 0.3956 & 0.0001 \\
& KW & 0.4017 & 0.0001 \\
& NH & 0.2929 & 0.6630 \\
& NR & 0.3651 & 0.0007 \\
& NK & 0.4266 & 0.0001 \\
& CW & 0.3313 & 0.0163 \\
\hline & & &
\end{tabular}

* HW: husk weight, NKR: mean number of kernels per row, $\mathrm{KW}$ : kernels weight, NH: number of husks, NR: number of rows, NK: total number of kernels, CW: cob weight.

Table VIII. Canonical roots extracted and $\chi^{2}$ tests corresponding to ear measurements associated with yield.

\begin{tabular}{lccccc}
\hline \multicolumn{2}{l}{ Hybrid type Roots } & Eigenvalue & $\chi^{2}$ & D. F. & $p<$ \\
\hline $2 \mathrm{X}$ & 1 & 4.260 & 282.960 & 28 & 0.0001 \\
& 2 & 1.226 & 128.576 & 18 & 0.0001 \\
& 3 & 0.528 & 54.146 & 10 & 0.0001 \\
& 4 & 0.172 & 14.743 & 4 & 0.0053 \\
$3 \mathrm{X}$ & 1 & 1.623 & 194.892 & 35 & 0.0001 \\
& 2 & 0.365 & 86.418 & 24 & 0.0001 \\
& 3 & 0.293 & 51.374 & 15 & 0.0001 \\
& 4 & 0.180 & 22.439 & 8 & 0.0042 \\
$4 \mathrm{X}$ & 5 & 0.035 & 3.816 & 3 & 0.282 \\
& 1 & 1.271 & 91.914 & 21 & 0.0001 \\
& 2 & 0.391 & 31.624 & 12 & 0.0016 \\
& 3 & 0.106 & 7.390 & 5 & 0.1933 \\
\hline
\end{tabular}


Table IX. Standardized coefficients for each canonical variate corresponding to ear measurements associated with yield*.

\begin{tabular}{|c|c|c|c|c|c|c|}
\hline Hybrid type & Variable & $\lambda_{1}$ & $\lambda_{2}$ & $\lambda_{3}$ & $\lambda_{4}$ & $\lambda_{5}$ \\
\hline $2 X$ & $\begin{array}{l}\text { HW } \\
\text { NKR } \\
\text { KW } \\
\text { NH } \\
\text { NR } \\
\text { NK } \\
\text { CW } \\
\text { Eigenvalue } \\
\text { Cumul. Perc. }\end{array}$ & $\begin{array}{r}0.273 \\
-0.244 \\
2.022 \\
-0.473 \\
-0.642 \\
-0.851 \\
-1.092 \\
4.260 \\
68.87\end{array}$ & $\begin{array}{r}0.601 \\
0.167 \\
1.849 \\
-0.364 \\
0.895 \\
-2.171 \\
-0.294 \\
1.226 \\
88.70\end{array}$ & $\begin{array}{r}-0.035 \\
-2.229 \\
0.511 \\
0.900 \\
-1.244 \\
2.150 \\
-0.185 \\
0.328 \\
97.22\end{array}$ & $\begin{array}{r}-1.237 \\
-1.951 \\
1.859 \\
-0.103 \\
-0.712 \\
1.415 \\
0.152 \\
0.172 \\
100.00\end{array}$ & $\begin{array}{l}- \\
- \\
- \\
- \\
- \\
-\end{array}$ \\
\hline $3 X$ & $\begin{array}{l}\text { HW } \\
\text { NKR } \\
\text { KW } \\
\text { NH } \\
\text { NR } \\
\text { NK } \\
\text { CW } \\
\text { Eigenvalue } \\
\text { Cumul. Perc. }\end{array}$ & $\begin{array}{r}1.023 \\
-0.661 \\
-0.574 \\
0.389 \\
-0.718 \\
0.788 \\
-0.102 \\
1.623 \\
65.01\end{array}$ & $\begin{array}{r}0.724 \\
-2.798 \\
0.122 \\
-0.772 \\
-1.155 \\
2.523 \\
0.679 \\
0.365 \\
79.63\end{array}$ & $\begin{array}{r}-0.451 \\
1.342 \\
-0.117 \\
0.660 \\
1.493 \\
-2.977 \\
1.430 \\
0.293 \\
91.41\end{array}$ & $\begin{array}{c}-0.651 \\
-2.441 \\
-1.282 \\
0.202 \\
-2.238 \\
3.527 \\
1.278 \\
0.180 \\
98.62\end{array}$ & $\begin{array}{r}0.002 \\
-1.545 \\
2.142 \\
0.044 \\
-1.092 \\
0.578 \\
-0.755 \\
0.035 \\
100.00\end{array}$ \\
\hline $4 X$ & $\begin{array}{l}\text { HW } \\
\text { NKR } \\
\text { KW } \\
\text { NH } \\
\text { NR } \\
\text { NK } \\
\text { CW } \\
\text { Eigenvalue } \\
\text { Cumul. Perc. }\end{array}$ & $\begin{array}{r}-0.269 \\
-0.899 \\
-2.086 \\
-0.063 \\
-1.193 \\
2.289 \\
1.027 \\
1.271 \\
0.719\end{array}$ & $\begin{array}{c}0.635 \\
0.063 \\
-0.788 \\
-0.281 \\
0.490 \\
0.721 \\
-0.373 \\
39.06 \\
94.02\end{array}$ & $\begin{array}{r}0.039 \\
0.592 \\
-0.656 \\
-0.199 \\
0.431 \\
-0.595 \\
-0.086 \\
0.106 \\
100.00\end{array}$ & $\begin{array}{l}- \\
- \\
- \\
- \\
- \\
-\end{array}$ & $\begin{array}{l}- \\
- \\
- \\
- \\
-\end{array}$ \\
\hline
\end{tabular}

* HW: husk weight, NKR: mean number of kernels per row, KW: kernels weight, NH: number of husks, NR: number of rows, NK: total number of kernels, CW: cob weight.

(Tab. X). Two canonical roots must be entered to take account of a significant part of the variation in the 3 groups (Tabs. XI and XII). In the case of 2X's a sort of "kernel shape" coefficient appears to be the meaning of the 1 st canonical root: $0.731 \cdot \mathrm{KL}-$ $0.855 \cdot \mathrm{KB}$ while a predominant variable appears in the constitution of the 2 first canonical roots in the case of $3 \mathrm{X}$ (KL and $\mathrm{KT}$ ) and $4 \mathrm{X}(\mathrm{KB}$ and $\mathrm{KL})$ hybrids.

\subsection{Genetic variance}

The group of $2 \mathrm{X}$ hybrids is also especially interesting because the variance among individual
Table X. Discriminant analysis with $\lambda$ Wilks tests and corresponding to measurements taken in kernels*.

\begin{tabular}{lccc}
\hline Hybrid type & Variable & Wilks $\lambda$ & $p<$ \\
\hline $2 \mathrm{X}$ & $\mathrm{KT}$ & 0.466 & 0.0310 \\
& $\mathrm{KL}$ & 0.619 & 0.0001 \\
& $\mathrm{~KB}$ & 0.630 & 0.0001 \\
$3 \mathrm{X}$ & $\mathrm{KT}$ & 0.789 & 0.0225 \\
& $\mathrm{KL}$ & 0.855 & 0.0005 \\
& $\mathrm{~KB}$ & 0.752 & 0.1808 \\
$4 \mathrm{X}$ & $\mathrm{KT}$ & 0.663 & 0.0781 \\
& $\mathrm{KL}$ & 0.741 & 0.0018 \\
& $\mathrm{~KB}$ & 0.727 & 0.0035 \\
\hline
\end{tabular}

\footnotetext{
* KT: kernel thickness, KL: kernel length, KB: kernel breadth.
} 
Table XI. Canonical roots extracted and $\chi^{2}$ tests corresponding to measurements taken in kernels.

\begin{tabular}{lccccc}
\hline Hybrid type Roots & Eigenvalue & $\chi^{2}$ & D. F. & $p<$ \\
\hline $2 \mathrm{X}$ & 1 & 0.792 & 83.192 & 12 & 0.0001 \\
& 2 & 0.315 & 27.757 & 6 & 0.0001 \\
& 3 & 0.019 & 1.753 & 2 & 0.4162 \\
$3 \mathrm{X}$ & 1 & 0.240 & 40.298 & 15 & 0.0004 \\
& 2 & 0.107 & 15.711 & 8 & 0.0467 \\
& 3 & 0.036 & 4.022 & 3 & 0.2591 \\
$4 \mathrm{X}$ & 1 & 0.328 & 37.885 & 9 & 0.0001 \\
& 2 & 0.244 & 16.475 & 4 & 0.0024 \\
\hline
\end{tabular}

Table XII. Standardized coefficients for each canonical variate corresponding to measurements taken in kernels*.

\begin{tabular}{llccc}
\hline Hybrid type & Variable & $\lambda_{1}$ & $\lambda_{2}$ & $\lambda_{3}$ \\
\hline $2 \mathrm{X}$ & KT & -0.159 & 0.649 & 0.851 \\
& KL & 0.731 & 0.841 & 0.005 \\
& KB & -0.855 & 0.411 & -0.427 \\
& Eigenvalue & 0.792 & 0.315 & 0.019 \\
& Cumul. Perc. & 70.38 & 98.35 & 100.00 \\
$3 \mathrm{X}$ & KT & -0.378 & -0.951 & -0.130 \\
& KL & -0.966 & 0.193 & 0.293 \\
& KB & 0.347 & -0.459 & 0.861 \\
& Eigenvalue & 0.240 & 0.107 & 0.036 \\
& Cumul. Perc. & 62.58 & 90.66 & 100.00 \\
$4 X$ & KT & -0.595 & -0.012 & - \\
& KL & 0.363 & 0.902 & - \\
& KB & -0.805 & 0.277 & - \\
& Eigenvalue & 0.327 & 0.244 & - \\
& Cumul. Perc. & 57.34 & 100.00 & -
\end{tabular}

* KT: kernel thickness, KL: kernel length, KB: kernel breadth.

plants within each hybrid is almost completely of an environmental nature. Assuming the construction has been properly performed, simple hybrids arise as a result of the intercrossing of 2 inbred lines. Thus, their genetic makeup is uniform.

In order to gain some help in the interpretation of multivariate results we have computed some values of variance components to assess the amount of the genetic component in these groups.
By means of an ANOVA Mixed Model [7] we have estimated the parameter $\sigma_{\mathrm{H}}^{2}$, i.e. the variance among single-cross hybrids. We have employed a mixed model because the HYBRID factor is random but the BLOCK factor is fixed. The mean square between simple hybrids estimate both components, $\sigma_{e}^{2}$ (the error component) and $n b \cdot \sigma_{\mathrm{H}}^{2}, n$ being the number of plants per block and $b$, the number of blocks. In the cases of $3 X$ and $4 X$ hybrids, in turn, the "Within Hybrids" component includes also a certain amount of genetic variation. In Table XIII the estimation of both components as well as the percentage of $\sigma_{\mathrm{H}}^{2}$ with respect to $\sigma_{\mathrm{TOT}}^{2}$ is presented. The BLOCK X TREATMENT interaction term was included in the TOTAL variance.

Table XIII. Estimation of the percentage of $\sigma_{\mathrm{H}}^{2}-$ the variance between simple hybrids - with respect to the total variance, $\sigma_{\mathrm{TOT}}^{2}$, corresponding to each trait*.

\begin{tabular}{lccc}
\hline & $\sigma_{\mathrm{H}}^{2}$ & $\sigma_{\text {TОт }}^{2}$ & $\%$ \\
\hline PH & 80.25 & 419.1 & 20 \\
DIAM1C & 0.029 & 0.197 & 15 \\
DIAM2C & 0.040 & 0.170 & 24 \\
DIAM1L & 0.030 & 0.230 & 13 \\
DIAM2L & 0.020 & 0.340 & 6 \\
EIH & 94.90 & 198.6 & 48 \\
EL & 0.964 & 11.99 & 8 \\
EBD & 0.098 & 0.239 & 41 \\
EMD & 0.180 & 0.308 & 58 \\
HW & 6.376 & 19.77 & 32 \\
NKR & 0.001 & 64.96 & 0 \\
KW & 750.3 & 3270.4 & 23 \\
NH & 1.610 & 3.258 & 49 \\
NR & 5.930 & 7.820 & 76 \\
NK & 11256 & 30196 & 37 \\
CW & 28.34 & 70.68 & 40 \\
KT & $33 \times 10^{-5}$ & $373 \times 10^{-5}$ & 9 \\
KL & $56 \times 10^{-4}$ & $195 \times 10^{-4}$ & 29 \\
KB & $185 \times 10^{-5}$ & $655 \times 10^{-5}$ & 28 \\
\end{tabular}

* PH: plant height, DIAM1C: upper minor stalk diameter, DIAM2C: lower minor stalk diameter, DIAM1L: upper major stalk diameter, DIAM2L: lower major stalk diameter, EIH: ear insertion height, EL: ear length, EBD: ear basal diameter, EMD: ear medium diameter, HW: husk weight, NKR: mean number of kernels per row, KW: kernels weight, $\mathrm{NH}$ : number of husks, NR: number of rows, NK: total number of kernels, $\mathrm{CW}$ : cob weight, KT: kernel thickness, KL: kernel length, KB: kernel breadth. 


\section{Discussion and conclusions}

\subsection{Plant architecture characteristics}

There is a decrease in discrimination from $2 \mathrm{X}$ hybrids toward $3 X$ 's and $4 X$; single hybrids show a greater magnitude of overall differences and to a greater extent. This may be observed in the decrease of significance of Wilks' tests for the most of the variables. Only EIH keeps its significance across the 3 groups and the differences among the stalk diameters nearly disappears. This may well be the consequence of many years of struggle against a very common problem in early materials: lodging. So, breeders have tended to rely on homogeneous materials with respect to stalk architecture traits. This is especially important because of the breeders' tendency to start breeding programs upon the base of hybrid populations, extracting from them the allelic background that may determine their high performances, and recombining to obtain new higher performances in the field. Breeders generally do not use landraces or wild relatives in their programs [13]. Double and three way hybrids merge the genetic makeup of the simple hybrids and lines they are based on, thus decreasing the magnitude of the differences among them. Also the number and the statistical significance of the roots removed are by far greater for $2 \mathrm{X}$ hybrids (Tab. II). Moreover, the variances among simple hybrids with respect to total variance (which may give a good idea of the amount of genetic variance) is not very high (Tab. XIII). Only EIH presented a relatively high value (48\%).

It would be worth remembering that canonical variables are absolutely correlation-free in the experiment. Thus, the inner variability partition calculated is much more than mere calculations of variances for further comparison with other populations. Indeed, we obtained discriminant functions explaining what the output of (maybe) years of genetic manipulation is. Canonical variates may be analyzed without any reference to other components and may be the starting point to take account of when considering the choice of selection variables.

\subsection{Ear architecture characteristics}

Given the results obtained for these traits, it may be said, - although the picture is not so clear - the situation here is not as threatening with respect to variation as in the plant architecture characteristics. The overall discrimination is not too low (Tab. IV) and the percentage of $\sigma_{\mathrm{H}}^{2}$ with respect to $\sigma_{\mathrm{TOT}}^{2}$ for the main variable (EMD) in the $2 \mathrm{X}$ group is high (58\%). There still seems to be room for selection without regard for reduction in variability. Anyway, it would be interesting to take measurements of more characteristics with respect to ear architecture in order to draw a more general conclusion.

\subsection{Ear measurements related to yield}

The discriminatory power diminishes, going from $2 X$ 's to $3 X$ and $4 X$ as observed in the case of plant traits. The distances between single-cross hybrids are greater than distances observed among the hybrids of the other groups ${ }^{1}$. There is an interesting degree of variation among simple hybrids and the percentages of $\sigma_{\mathrm{H}}^{2}$ with respect to $\sigma_{\text {TOT }}^{2}$ are relatively high (Tab. XIII) - around $40 \%$ - and this is especially interesting taking into account the great manipulation these traits have undergone through years of breeding towards the highest possible yield: it seems that variability is still high.

\subsection{Kernel measurements}

With the exception of $\mathrm{KB}$ in the $3 \mathrm{X}$ group, the general discrimination is at the same statistical level of the characteristics associated with yield. Especially for KL the distances between hybrids within each group are statistically significant and this variable is also relevant as canonical root component in all three groups.

\footnotetext{
${ }^{1}$ We have computed and statistically tested squared Mahalanobis distances [Seber, 1984] among hybrids but we have not included them here, for the sake of brevity.
} 


\section{Concluding remarks}

This survey may be extended to many different characteristics other than the morphological ones. Metabolic as well as chemical-composition or even molecular characteristics would be worth analyzing. Considering more inclusive levels of complexity, populational or ecological characteristics must be included especially addressing the ecological impact that the ever-increasing-uniformity breeding programs and the insertion of foreign genes can have on agro-ecosystems.

Acknowledgements: This work was supported with funds from the UBACyT project TG 002 (UBA).

\section{Appendix}

Canonical variates can be obtained from the original data matrix $\mathrm{Y}(n \cdot p$, where $n$ is the number of plants measured and $p$ is the number of variables) through a linear transformation [Seber, 1984] that maximizes the differences among populations and minimize the variation within them. The linear transformation:

$$
\mathrm{Z}=\mathrm{Y} \cdot \mathrm{T}
$$

(where $\mathrm{T}$ is the $p \cdot p$ transformation matrix) can be found by solving the characteristic equation:

$$
|\mathrm{B}-\alpha \cdot \mathrm{W}|=0
$$

where $\mathrm{B}$ and $\mathrm{W}$ are between-groups and the within-groups sum of squares and products matrices, respectively. This equation has $p$ eigenvalues $\left(\alpha_{1}\right.$, $\alpha_{2}, \ldots, \alpha_{p}$ ) given $p$ is the number of original variables measured. Hence, the eigenvectors $t_{i}$ corresponding to each of the canonical roots $\alpha_{\mathrm{i}}$, can be extracted.

Thus $\mathrm{Z}$ is an $n \cdot p$ matrix of fully orthogonal transformed variables maximizing the variation among the populations.

\section{References}

[1] Anagnostakis S.C., Biological control of chestnut blight, Science 215 (1982) 466-471.
[2] Brown A.H.D., Weir B.S., Measuring genetic variability in plant populations, in: Tanksley S.D., Orton T.J. (Eds.), Isozymes in Plant Genetics and Breeding, Amsterdam, Elsevier, 1983.

[3] Camussi A., Ottaviano E., Calinski T., Kaczmarek Z., Genetic distances based on quantitative traits, Genetics 111 (1985) 945-962.

[4] Duvick D.N., Genetic diversity in major farm crops on the farm and in reserve, Econ. Bot. 38 (1984) $161-178$.

[5] Duvick D.N., Sources of genetic advances for the future, in: Burris J.S. (Ed.), Proc. Ninth Ann. Seed Tech. Conf., Ames, Iowa, Seed Science Center, 1987.

[6] Duvick D.N., Genetic contributions to advances in yield of US maize, Maydica 37 (1992) 69-79.

[7] Falconer D.S., Mackay T., Introduction to Quantitative Genetics, 2nd ed., Longman, London, 1996.

[8] Felsenstein J., Numerical methods for inferring evolutionary trees, Q. Rev. Biol. 57 (1982) 379-404.

[9] Fowler C., Mooney P., Shattering: Food, Politics and the loss of genetic diversity, Tucson, Arizona, University of Arizona Press, 1990.

[10] Goodman M.M., Genetic distances: measuring dissimilarity among populations, Yearb. Phys. Anthropol. 17 (1973) 1-38.

[11] Gottlieb L.D., Genetic and morphological evolution in plants, Am. Nat. 123 (1984) 681-709.

[12] Marshall D.R., Limitations to the use of germplasm collections, in: Brown A., Frankel O., Marshall D.R., Williams J.T. (Eds.), The use of Plant genetic resources, New York, Cambridge Univ. Press, 1989.

[13] Peeters J.P., Galwey N.W., Germplasm collections and breeding needs in Europe, Econ. Bot. 42 (1988) 503-521.

[14] Seal H.L., Multivariate statistical analysis for biologists, Methuen, London, 1964.

[15] Seber G., Multivariate observations, Wiley, New York, 1984.

[16] Whitehouse R., An application of canonical analysis to plant breeding, Genet. Agrar. 23 (1969) 61-69. 